\title{
Semi-annual oscillations in Saturn's low-latitude stratospheric temperatures
}

\author{
Glenn S. Orton ${ }^{1}$, Padma A. Yanamandra-Fisher ${ }^{1}$, Brendan M. Fisher ${ }^{2}$, A. James Friedson ${ }^{1}$, Paul D. Parrish ${ }^{3}$, \\ Jesse F. Nelson ${ }^{4}$, Amber Swenson Bauermeister ${ }^{5}$, Leigh Fletcher ${ }^{1}$, Daniel Y. Gezari ${ }^{6}$, Frank Varosi ${ }^{7}$, \\ Alan T. Tokunaga ${ }^{8}$, John Caldwell ${ }^{9}$, Kevin H. Baines ${ }^{2}$, Joseph L. Hora ${ }^{10}$, Michael E. Ressler ${ }^{11}$, Takuya Fujiyoshi ${ }^{12}$, \\ Tetsuharu Fuse ${ }^{12}$, Hagop Hagopian ${ }^{13}$, Terry Z. Martin ${ }^{1}$, Jay T. Bergstralh ${ }^{14}$, Carly Howett ${ }^{15}$, William F. Hoffmann ${ }^{16}$, \\ Lynne K. Deutsch $\ddagger$, Jeffrey E. Van Cleve ${ }^{17}$, Eldar Noe ${ }^{18}$, Joseph D. Adams ${ }^{19}$, Marc Kassis ${ }^{20}$ \& Eric Tollestrup ${ }^{21}$
}

\begin{abstract}
Observations of oscillations of temperature and wind in planetary atmospheres provide a means of generalizing models for atmospheric dynamics in a diverse set of planets in the Solar System and elsewhere. An equatorial oscillation similar to one in the Earth's atmosphere ${ }^{1,2}$ has been discovered in Jupiter ${ }^{3-6}$. Here we report the existence of similar oscillations in Saturn's atmosphere, from an analysis of over two decades of spatially resolved observations of its $7.8-\mu \mathrm{m}$ methane and $12.2-\mu \mathrm{m}$ ethane stratospheric emissions, where we compare zonal-mean stratospheric brightness temperatures at planetographic latitudes of $3.6^{\circ}$ and $15.5^{\circ}$ in both the northern and the southern hemispheres. These results support the interpretation of vertical and meridional variability of temperatures in Saturn's stratosphere ${ }^{7}$ as a manifestation of a wave phenomenon similar to that on the Earth and in Jupiter. The period of this oscillation is $14.8 \pm 1.2$ terrestrial years, roughly half of Saturn's year, suggesting the influence of seasonal forcing, as is the case with the Earth's semi-annual oscillation ${ }^{1}$.
\end{abstract}

These conclusions are based on a sequence of filtered mid-infrared maps or images of Saturn, through narrow- to medium-band spectral filters that are sensitive to upwelling radiance emerging from Saturn's stratosphere. As in our study of Jupiter ${ }^{6}$, we preferred to use the emission of stratospheric methane at wavelengths of around $7.8 \mu \mathrm{m}$ to detect the stratospheric temperature field near the 20 -mbar pressure level in the atmosphere, because methane is expected to be well mixed in Saturn's stratosphere. Thus, all variations in the thermal radiance must be attributed to variations in temperature, rather than in the methane abundance. However, because $7.8-\mu \mathrm{m}$ methane emission is much fainter for Saturn than it is for Jupiter, most of our earliest observations with lengthy raster scans consist only of observations of much brighter stratospheric emission from ethane at wavelengths of around $12.2 \mu \mathrm{m}$ (see the Supplementary Information), because only these images had sufficient signal-to-noise ratios to be useful. Figure 1 shows examples of $7.8-\mu \mathrm{m}$ methane emission observed from the NASA Infrared Telescope Facility (IRTF) in two different phases of the oscillation. Details of the observations are given in the Supplementary Information.

The angular resolution of scans and images at the IRTF was limited by diffraction to no better than $0.7 \operatorname{arcsec}$ (at latitude $4^{\circ}$ ) for $7.8-\mu \mathrm{m}$ methane emission and $1.1 \operatorname{arcsec}\left(\right.$ at latitude $7^{\circ}$ ) for $12.2-\mu \mathrm{m}$ ethane emission, with some additional blurring arising from seeing (that is, distortion due to terrestrial atmospheric turbulence). (Here and below, latitude values without an explicit attribution refer to either the northern or the southern hemisphere.) It is possible to resolve differences between emission at planetographic latitudes of $3.6^{\circ}$ and $15.5^{\circ}$ (planetocentric latitudes of $3.0^{\circ}$ and $13.0^{\circ}$ ) in all the images used in this study, which is a requirement for this investigation. We ignored regions of the planet that were potentially obscured by the rings, defined for this purpose as extending from $74,500 \mathrm{~km}$ (the inner edge of the $\mathrm{C}$ ring) out to $154,000 \mathrm{~km}$ (the outer edge of the A ring) from the centre of the planet. We did not consider images that were sufficiently close to ring-plane crossing times that substantial ring obscuration of the atmosphere at latitude $3.6^{\circ}$ might occur. Potential small obscurations were included in the uncertainties associated with the 1997 data (see the Supplementary Information). Brightness temperatures were averaged over $\pm 15^{\circ}$ of longitude around the central meridian, minimizing limb-brightening effects while maximizing the latitude extent of our sample. Figure 2 shows representative plots of zonally averaged brightness temperature versus latitude for methane and ethane for each year of observation.

In Figs 1 and 2 there is clear evidence of low-latitude oscillations between near-equatorial and higher latitudes, corresponding to the differences seen in the vertical structures observed by the Cassini Composite Infrared Spectrometer $(\mathrm{CIRS})^{7}$. These differences are maximized by selecting brightness temperatures at latitude $3.6^{\circ}$ $\left(3.0^{\circ}\right.$ planetocentric) north or south and subtracting temperatures at latitude $15.5^{\circ}\left(13.0^{\circ}\right.$ planetocentric) in the same hemisphere. Measurements of brightness temperatures at latitude $3.6^{\circ}$ north or

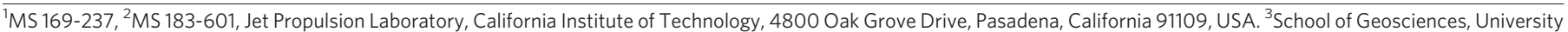

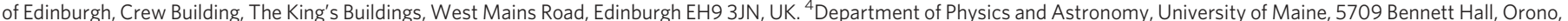

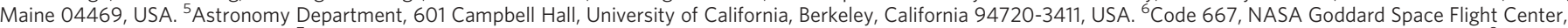

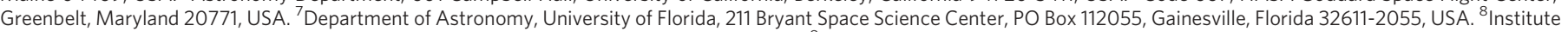

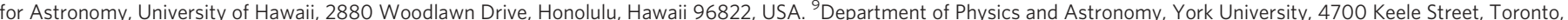

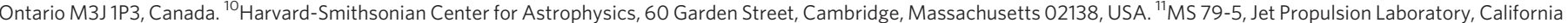

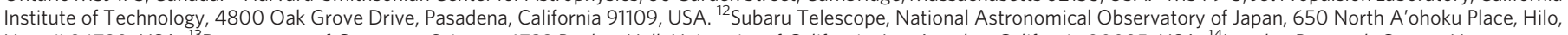
Hawaii 96720, USA. ${ }^{13}$ Department of Computer Science, 4732 Boelter Hall, University of California, Los Angeles, California 90095, USA. ${ }^{14}$ Langley Research Center, Hampton, Virginia 23681-2199, USA. ${ }^{15}$ Atmospheric, Oceanic and Planetary Physics, Clarendon Laboratory, Oxford University, Parks Road, Oxford, Oxfordshire OX1 3PU, UK. ${ }^{16}$ Steward

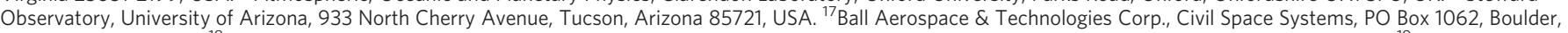
Colorado 80306, USA. ${ }^{18}$ MS 183-501, Jet Propulsion Laboratory, California Institute of Technology, 4800 Oak Grove Drive, Pasadena, California 91109, USA. ${ }^{19}$ Department of

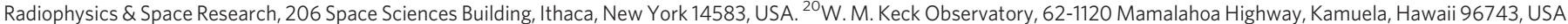
${ }^{21}$ NASA Infrared Telescope Facility, Institute for Astronomy, 640 North A'ohoku Place, Hilo, Hawaii 96822, USA. †Deceased 
south sample almost the same vertical temperature structure as do measurements directly at the equator ${ }^{7}$, but this selection provides a significantly larger number of samples that are not precluded by potential ring obscuration of the equator itself.

In Figure 3 we plot these differences as a function of time, which makes clear the cyclic nature of the temperature differences between these latitudes over the past 22 years. The variability of equatorial emission between Voyager 1 observations in 1980 and the 1989 IRTF observations $^{8}$ is consistent with this periodicity. Figure 3 also validates our first-order assumption that the oscillation is essentially equal in each hemisphere. Fitting the ethane emission data, which cover more than one cycle, results in a 'peak-to-peak' amplitude of just over $2 \mathrm{~K}$ in the $12.2-\mu \mathrm{m}$ brightness temperature difference variation $(8 \mathrm{~K}$ at $7.8 \mu \mathrm{m})$ and a period of $14.8 \pm 1.2 \mathrm{yr}$.

We did not attempt to derive stratospheric kinetic temperatures using our measured brightness temperatures, unlike in the investigation of Jupiter's stratospheric temperature variability ${ }^{6}$ with similar data, primarily because of the intractability of separating latitudinal variations of stratospheric temperatures from ethane abundances. However, the altitude and latitude variability of temperatures derived by the Cassini CIRS experiment ${ }^{7}$ provides a sound basis for the latitudinal variability of the temperature and ethane vertical profiles in

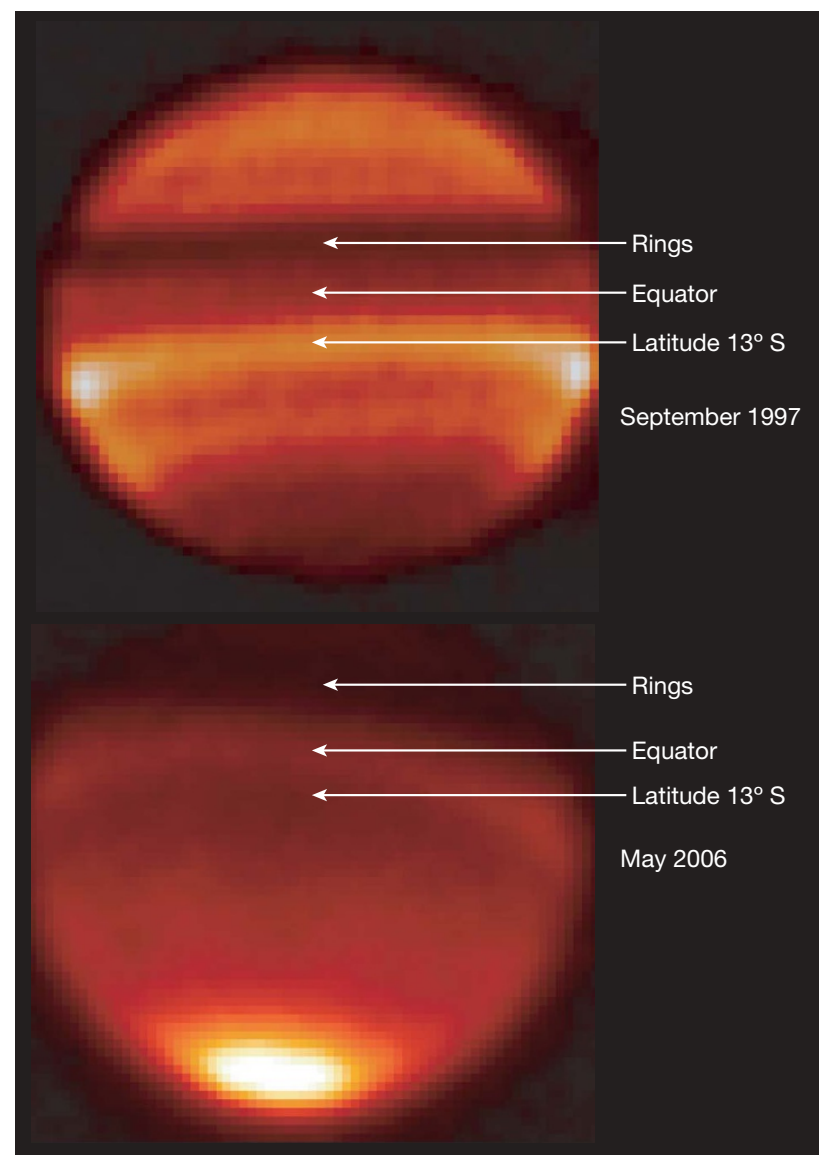

Figure 1 | Representative thermal images of Saturn showing two phases of the oscillation. Images showing $7.8-\mu \mathrm{m}$ methane emission from Saturn's stratosphere taken at the IRTF, illustrating the alternating amplitudes of low-latitude temperatures in the 20 -mbar altitude range between the equator and latitude $15^{\circ} \mathrm{S}$. At this wavelength, emission from Saturn's rings is lower than that from the planet, and their primary effect is to obscure thermal emission from the atmosphere. These images demonstrate Saturn's appearance during a relative minimum of equatorial temperatures (1997) and during a relative maximum of equatorial temperatures (2006). A similar equatorial brightening was noted in comparing IRTF images from 1989 with low equatorial temperatures derived from the Voyager-1 Infrared Radiometer Interferometer Spectrometer experiment ${ }^{8}$. We note that the warm south pole in May 2006 is driven by seasonal variability ${ }^{10}$. the 2005-2006 epoch. Figure 4 shows that the correlation of the spacecraft results with our 2006 observations is extremely good, if the Cassini CIRS results are appropriately blurred to match the average spatial resolution of the ground-based data. This implies that the amplitude of the actual variations is much higher in the atmosphere than is indicated in Fig. 3. Figure 4 also shows that the observed variability in brightness temperature difference between 2003 and 2007, which far exceeds the errors associated with the individual measurements, may arise simply from variations of atmospheric seeing conditions.

The $\sim 15$-yr period we derive is considerably longer than the two- to four-year cycles associated with the Earth's quasi-biennial

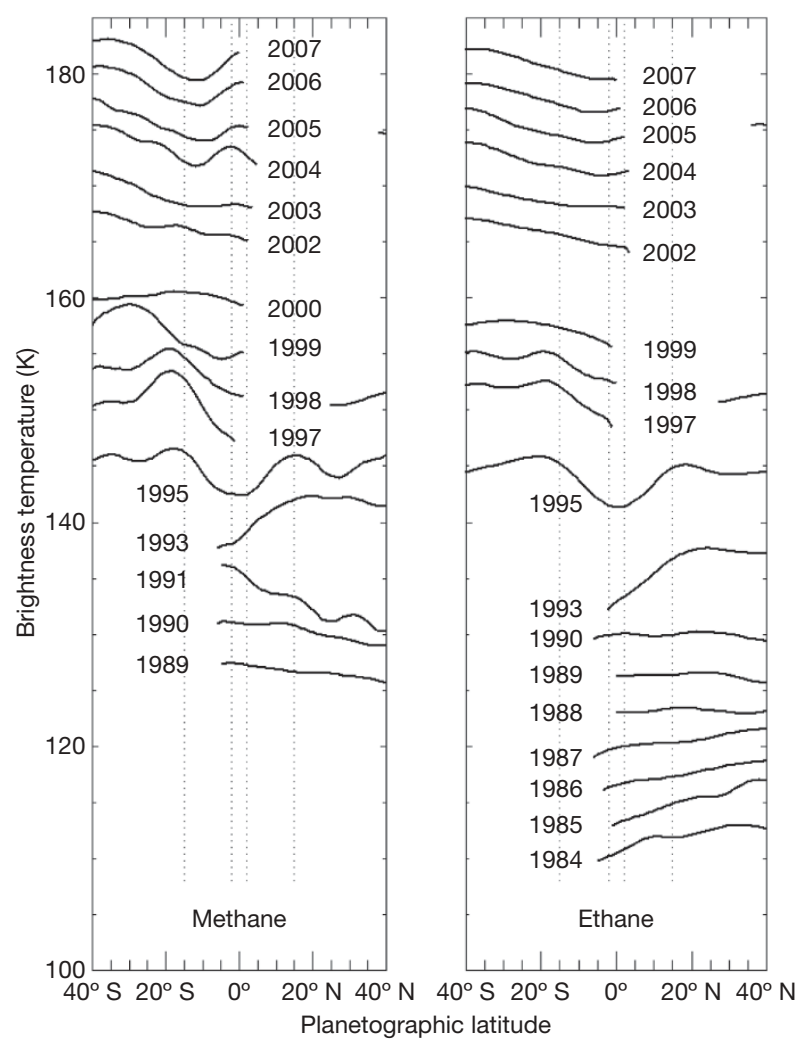

Figure 2 | Representative plots of zonally averaged brightness

temperatures versus time. Methane emission at wavelengths of around $7.8 \mu \mathrm{m}$ is shown in the left panel and ethane emission at wavelengths of around $12.2 \mu \mathrm{m}$ is shown in the right panel. The methane brightness temperature plot is correct for 1989 and offset by $+3 \mathrm{~K}$ per year thereafter. The ethane plot is correct for 1984 and offset by $+3 \mathrm{~K}$ per year thereafter. The dotted lines denote latitudes of $3.6^{\circ}$ and $15.5^{\circ}$ north and south, for reference; these are the two reference latitudes used (per hemisphere) to plot the brightness temperature differences shown in Fig. 3. Most of the observations were made at the IRTF. Single-element IRTF facility photometers were used to create raster-scanned maps of Saturn between 1985 and 1990 in a way similar to stratospheric maps of Jupiter ${ }^{6}$. The first two-dimensional thermal infrared imaging camera used at the IRTF observed both methane and ethane emission from Saturn ${ }^{8}$ in 1989; we included additional data from this camera taken in 1988, 1990 and 1991. Other two-dimensional cameras used to collect data in subsequent years include MIRAC ${ }^{11}$, MIRLIN ${ }^{12}$, and MIRSI ${ }^{13}$; these data include 1993 MIRAC observations from the 2.3-m Steward Observatory Bok Telescope. Until 2003 , Saturn was observed only as a target of opportunity during observation runs primarily dedicated to providing ground-based support for the Galileo mission at Jupiter. Beginning in 2003, Saturn became a primary target, with a concomitant increase in observation time and a higher signalto-noise ratio characterizing each image. We also included data from larger telescopes with improved diffraction-limited angular resolution: images of both methane and ethane emission taken using MIRLIN on the 10-m Keck I Telescope, an image of methane emission taken on the Keck II Telescope using the same facility's long-wavelength spectrometer ${ }^{10}$, and an image of ethane emission taken on the 8.2-m Subaru Telescope. 


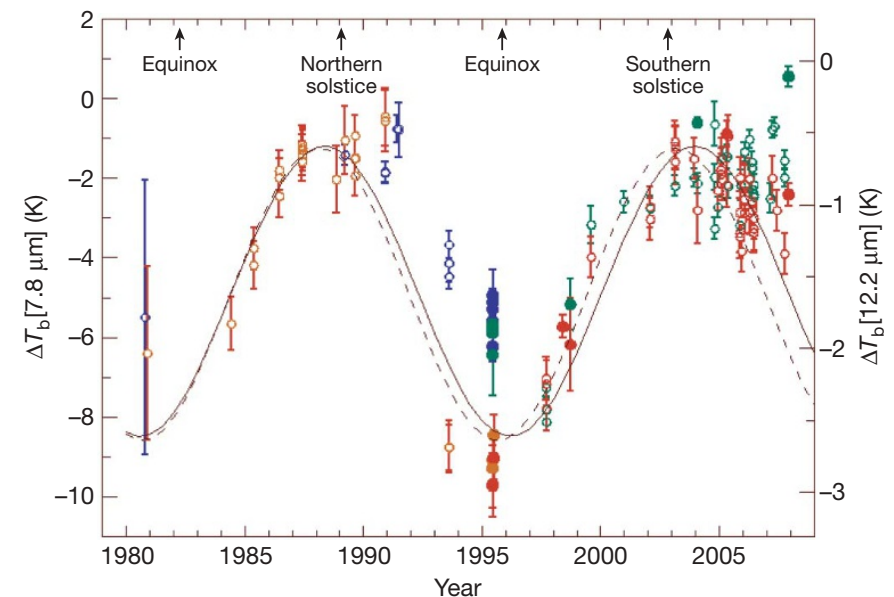

Figure 3 | Difference of zonally averaged brightness temperatures at two latitudes versus time. The planetographic latitudes are $3.6^{\circ}$ and $15.5^{\circ}$ north and south, and the plot is given for spectral regions representing methane emission $(\sim 7.8 \mu \mathrm{m})$ and ethane emission $(\sim 12.2 \mu \mathrm{m})$, showing one-s.d. uncertainties. Peak values represent brightness temperatures higher at latitude $3.6^{\circ}$ than at latitude $15.5^{\circ}$. Open circles represent data taken at the IRTF or the Bok Telescope and filled circles represent data taken at Palomar Observatory (1995), at the W. M. Keck Observatory $(1998,2004)$ or using the Subaru Telescope $(2005,2007)$. Methane brightness temperature differences between $3.6^{\circ} \mathrm{N}$ and $15.5^{\circ} \mathrm{N}$ are denoted by blue symbols, and those between $3.6^{\circ} \mathrm{S}$ and $15.5^{\circ} \mathrm{S}$, by green symbols. Ethane brightness temperature differences between $3.6^{\circ} \mathrm{N}$ and $15.5^{\circ} \mathrm{N}$ are denoted by orange symbols, and those between $3.6^{\circ} \mathrm{S}$ and $15.5^{\circ} \mathrm{S}$, by red symbols. Despite evidence for the non-uniform distribution of ethane with latitude ${ }^{7}$, a high correlation between the variations in brightness temperature differences at $7.8 \mu \mathrm{m}$ and $12.2 \mu \mathrm{m}$ exists for all years (except 1993 and 1995); we find that $\Delta T_{\mathrm{b}}[12.2 \mu \mathrm{m}]=2.5 \Delta T_{\mathrm{b}}[7.8 \mu \mathrm{m}]+2.71 \mathrm{~K}$, allowing us to plot the two sets of data as shown. The difference in $\Delta T_{\mathrm{b}}$ values can be attributed to the different vertical regions sampled by the two different wavelengths. A sinusoidal function that best fits the individual data (solid line) yields a period of $15.6 \mathrm{yr}$; a similar sinusoidal function that best fits an annual average of the data (dashed line) yields a period of $15.0 \mathrm{yr}$. The true time dependence is clearly not a simple sinusoid, and a correlation between the 1985-1992 12.2$\mu \mathrm{m}$ data and 1997-2007 data yields a period of $13.9 \mathrm{yr}$. The mean of the three calculated periods is $14.8 \pm 1.2 \mathrm{yr}$, which we take as our fitted value. The 1980 points, taken from convolutions of appropriate filter functions over Voyager-1 Infrared Radiometer Interferometer Spectrometer spectra from a north-south mapping sequence, were not considered in the fit of the sinusoidal function, although they are consistent with it.

oscillation $^{1,2}$ or Jupiter's quasi-quadrennial oscillation ${ }^{3-5}$. In fact, it is roughly one-half of Saturn's 29.4-yr orbital period, which raises the possibility that, like the Earth's semi-annual oscillation ${ }^{1}$, these oscillations are phase locked by seasonal changes. In the Earth's semiannual oscillation, westerly acceleration of the zonal wind is due, at least in part, to absorption of upwardly propagating, high-speed Kelvin waves, whereas easterly acceleration is associated with a combination of forcing by the seasonally reversing mean meridional circulation and seasonal momentum transfer from planetary waves in the winter hemisphere. Similar processes may be occurring on Saturn. Continued observations from the ground in the next few years and by the Cassini CIRS experiment during the operations extended beyond the July 2008 end of the primary mission should confirm both their periodicity and how they affect the spatial wave structure during a time when a rapid return to cooler equatorial brightness temperatures is expected.

Theoretical models similar to those for Jupiter's quasi-quadrennial oscillation ${ }^{4,9}$ must be applied to the spatial and temporal variabilities we have observed in Saturn to determine the influence on the stratosphere of perturbations of the troposphere and radiative heating, including the influence of shadowing by the rings. Such models will

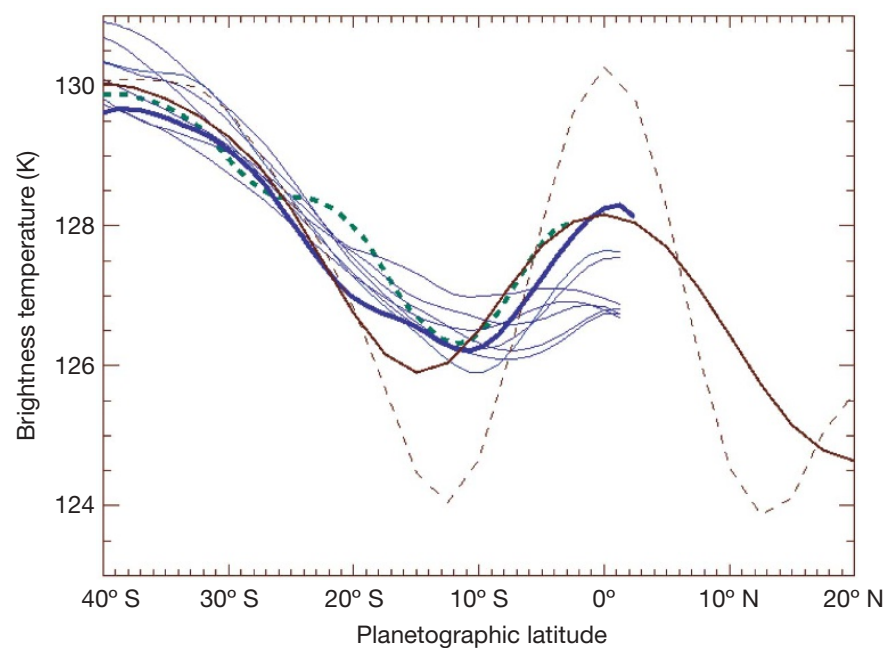

Figure 4 | Comparison of zonally averaged measurements and simulations of the meridional variability. Measurements are from 2006 (blue lines) and the simulated brightness temperatures are derived from convolving the upwelling radiance over the $7.8-\mu \mathrm{m}$ filter used in the measurements. The thick blue line represents the observation with the best seeing, and the thick dashed green line represents higher resolution 2004 observations ${ }^{10}$, for comparison. The upwelling radiance simulations (dashed brown line) are based on the zonal-mean temperature structures derived from Cassini CIRS spectra of Saturn's limb ${ }^{7}$. They have been convolved with a typical seeing to simulate observing conditions more accurately (solid brown line). It is most probable that the apparent variability in the observations arises from changes of seeing conditions from observation to observation; the best image of 2006 is plotted (thick blue line) for comparison. These changes are also likely to contribute to the ostensible short-term variability of the brightness temperature differences in Fig. 3.

allow us to determine the extent to which phenomena similar to Earth's quasi-biennial oscillation may be responsible for latitudinal transport ${ }^{7}$. Identifying analogous phenomena would have wide implications for transport in the stratospheres of all outer planets.

Received 11 December 2007; accepted 7 March 2008.

1. Andrews, D. G., Holton, F. R. \& Leovy, C. B. Middle Atmosphere Dynamics (Academic, New York, 1987).

2. Baldwin, M. et al. The quasi-biennial oscillation. Rev. Geophys. 39, 179-230 (2001).

3. Leovy, C. B., Friedson, A. J. \& Orton, G. S. The quasiquadrennial oscillation of Jupiter's equatorial stratosphere. Nature 354, 380-382 (1991).

4. Friedson, A. J. New observations and modeling of a QBO-like oscillation in Jupiter's stratosphere. Icarus 137, 34-55 (1999).

5. Flasar, F. M. et al. An intense stratospheric jet on Jupiter. Nature 427, 132-135 (2004).

6. Orton, G. S. et al. Thermal maps of Jupiter: Spatial organization and time dependence of stratospheric temperatures, 1980-1991. Science 252, 537-542 (1991).

7. Fouchet, T. et al. An equatorial oscillation in Saturn's middle atmosphere. Nature doi:10.1038/nature06912 (this issue).

8. Gezari, D. Y. et al. New features in Saturn's atmosphere revealed by highresolution thermal infrared images. Nature 342, 777-780 (1989).

9. Li, X. \& Read, P. L. A mechanistic model of the quasi-quadrennial oscillation in Jupiter's stratosphere. Planet. Space Sci. 48, 637-669 (2000).

10. Orton, G. S. \& Yanamandra-Fisher, P. A. Saturn's temperature field from highresolution middle-infrared imaging. Science 307, 696-701 (2005).

11. Hoffman, W. F., Fazio, G. G., Shivanandan, K., Hora, J. L. \& Deutsch, L. K. in Infrared Detectors and Instrumentation (Proc. SPIE 1946) (ed. Fowler, A. M.) 449-460 (SPIE, 1993).

12. Ressler, M. E., Werner, M. E., Van Cleve, J. \& Chou, H. A. The JPL deep-well midinfrared array camera. Exp. Astron. 3, 277-280 (1994).

13. Deutsch, L. K., Hora, J. L., Adams, J. D. \& Kassis, M. in Instrument Design and Performance for Optical/Infrared Ground-based Telescopes (Proc. SPIE 4841) (eds Iye, M. \& Moorwood, A. F. M.) 106-116 (SPIE, 2003).

Supplementary Information is linked to the online version of the paper at www.nature.com/nature.

Acknowledgements We acknowledge help from the staffs of the NASA IRTF, the Hale Observatories, the W. M. Keck Observatory, the Subaru Telescope and the 
Steward Observatory for making these observations possible, as well as support for JPL from the Cassini project and NASA research and analysis programs. P.D.P. was a NASA Postdoctoral Fellow; L.F. was supported by the NASA Postdoctoral Program; J.F.N., A.S.B. and E.N. were Caltech Summer Undergraduate Research Fellows; and J.F.N. was supported by the NASA Space Grant program at JPL during the course of this work. The radiative-transfer calculations were performed on JPL supercomputer facilities. Full acknowledgments are given in the Supplementary Information section.

Author Contributions G.S.O., P.A.Y.-F., B.M.F., A.J.F., P.D.P., L.F., D.Y.G., F.V., A.T.T., J.C., K.H.B., J.L.H., M.E.R., J.L.H., Ta.F., Te.F., T.Z.M., J.T.B., W.F.H., L.K.D.,
J.E.V.C. and C.H. made the observations. J.L.H., J.D.A., M.K. and E.T. built and adapted the MIRSI instrument to routine operations at the IRTF. G.S.O., P.A.Y.-F., B.M.F., P.D.P., J.F.N., A.S.B., E.N., D.Y.G. and F.V. reduced the data. G.S.O., J.F.N. and A.S.B. analysed the data for time dependence and J.F.N. provided initial sinusoidal fits to the data. G.S.O. performed the radiative-transfer calculations, made the final data selection, and derived the final fitted parameters. G.S.O. and A.J.F. wrote the initial draft. All authors discussed the results and commented on the manuscript.

Author Information Reprints and permissions information is available at www.nature.com/reprints. Correspondence and requests for materials should be addressed to G.S.O. (glenn.orton@jpl.nasa.gov). 